\title{
Melanoma del conducto auditivo externo. Presentación de un caso
}

Melanoma of the external auditive canal. Case report

\section{Pedro Rosales Torres ${ }^{1}$, Rafael Pila Pérez ${ }^{2}$, Pedro León Acosta ${ }^{3}$, Rafael Pila Peláez ${ }^{4}$, Jaider Luis Saurith Monterrosa ${ }^{5}$}

1. Especialista de $1 .^{\text {er }}$ grado en Anatomía Patológica. Instructor

2. Especialista de $2 .^{\circ}$ grado en Medicina Interna. Profesor Titular

3. Especialista de $1 .{ }^{\text {er }}$ grado en Medicina Interna. Instructor

4. Especialista de $2 .^{\circ}$ grado en Medicina Interna. Profesor Auxiliar

5. Especialista de $1 .^{\text {er }}$ grado en Medicina Interna

\section{RESUMEN}

Los melanomas cutáneos son neoplasias malignas cuya incidencia ha aumentado marcadamente en las últimas cinco décadas. En cuanto a los melanomas cutáneos primarios, la localización en cabeza y cuello representa el 17\%. De estos, entre el $7 \%$ y el 16\% se asientan en el oído externo, de los cuales, aproximadamente el 60\% ocurren en el hélix y el antihélix ${ }^{(1,2)}$. El compromiso del conducto auditivo externo rara vez es informado y cuando ocurre, tiene lugar en el meato.

Reportamos el caso de un paciente campesino con exposición a la luz solar de larga data. El paciente presentó una lesión de $1 \times 1,5 \mathrm{~cm}$, aproximadamente, de color pardo oscuro, en el conducto auditivo externo, con otalgia, otorrea con presencia de pus, sangre e hipoacusia y adenopatía preauricular y cervical. El diagnóstico se confirmó con punción por aspiración fina (PAFF) del tumor y, además, con el estudio de la pieza postoperatoria, que confirmó el resultado de la PAFF.

PALABRAS CLAVE: maligno, melanoma, conducto auditivo externo, HMB45 .

\section{SUMMARY}

Cutaneous melanomas are malignant neoplasms whose incidence has increased markedly in the last five decades. Among the primary cutaneous melanomas, the head and neck location represent $17 \%$. Of these, 7 to $16 \%$ settle in the outer ear, of which about $60 \%$ occur in the helix and anti-helix. External auditory canal involvement is rarely reported and when it occurs, it occurs in the meatus.

We report the case of a peasant patient with long-term exposure to sunlight. He presented a lesion of approximately $1 \times 1.5 \mathrm{~cm}$ in the dark brown external audi-

\section{Correspondencia:}

Jaider Luis Saurith Monterrosa

Email:

jaidersaurith@hotmail.com

Recibido: 23/05/19

Aceptado: 05/08/19

Conflictos de interés:

No se reportan conflictos de interés.

\section{Financiación:}

Ninguna. 
tory canal with otalgia, otorrhea with the presence of pus, blood accompanied by hearing loss and preatrial and cervical adenopathy, whose diagnosis was confirmed by fine aspiration puncture (PAFF) of the tumor in addition to the study of the postoperative piece that confirmed the result of the PAFF.

KEY WORDS: Malignant, melanoma, external auditory canal, HMB-45.

\section{INTRODUCCIÓN}

Los tumores malignos del conducto auditivo externo (CAE) son poco frecuentes. Su baja incidencia y la presentación clínica similar a los procesos crónicos del oído pueden implicar retraso en el diagnóstico ${ }^{(3)}$.De todos los tumores que afectan el oído, el 60\% se encuentra en la región auricular, el $28 \%$ en el CAE y el $2 \%$ en el oído medio ${ }^{(4)}$. Los melanomas de oído externo constituyen menos del $1 \%$ de todos los melanomas cutáneos y se asientan, fundamentalmente, en el pabellón auricular ${ }^{(2)}$. Otros autores señalan al melanoma como el responsable del $0,5 \%$ de las neoplasias que se asientan en el oído externo ${ }^{(4-6)}$. Las neoplasias en esta localización constituyen el 7\%-14,5\% de los melanomas de cabeza y cuello ${ }^{(4)}$. El hallazgo de tumores melánicos originados en el CAE es raro ${ }^{(4)}$. Se presentar el caso de un paciente con melanoma del CAE, el primero diagnosticado en nuestro hospital en 55 años.

\section{CASO CLÍNICO}

Paciente de 60 años, masculino, fototipo III según la escala Fitzpatrick, campesino dedicado a las labores agrícolas, sin antecedentes familiares o personales de interés. Refirió otalgia izquierda, otorrea con discreta cantidad de pus y sangre de 6 meses de evolución, acompañada de hipoacusia de 3 meses. Se nota, además, tumefacción preauricular en la cadena lateral izquierda. Oído derecho sin alteraciones.

En la exploración se aprecia adenopatía preauricular izquierda, no dolorosa, de $2 \mathrm{~cm}$ y fija y varias adenopatías en número de tres, con un tamaño de 1 a $2 \mathrm{~cm}$, de consistencia pétrea, no dolorosas y fijas, sin reportarse otras localizaciones en el cuello y las fosas supraclaviculares.

En la otoscopia se observó el CAE ocupado por un tumor de aspecto exofítico, que obstruye dicho conducto de $1 \times 1,2 \mathrm{~cm}$, aproximadamente, duro, de color pardo oscura y fácilmente sangrante (figura 1).

Radiografía de tórax, cráneo y serie radiológica de huesos largos, ultrasonido abdominal, tomografía axial computarizada (TAC) de cráneo, mediastino y retroperitoneo, todas normales. Se practica TAC de columna cervical y oídos en la que se encuentra ocupación de CAE por una masa isodensa de $1 \times 0,9 \mathrm{~cm}$, sin apreciarse restos timpánicos ni de huesecillos. Parótida sin alteraciones. Adenopatías preauriculares de $1,5 \times 2 \mathrm{~cm}$ y varias en la cadena lateral izquierda del cuello, de 1 a $2 \mathrm{~cm}$. La punción por aspiración con aguja fina (PAFF) de la adenopatía preauricular informa la presencia de un melanoma, al igual que la PAFF del tumor.

El paciente fue sometido a intervención quirúrgica con vaciamiento ganglionar radical de oído izquierdo y cervical con parotidectomía superficial. El informe opera-

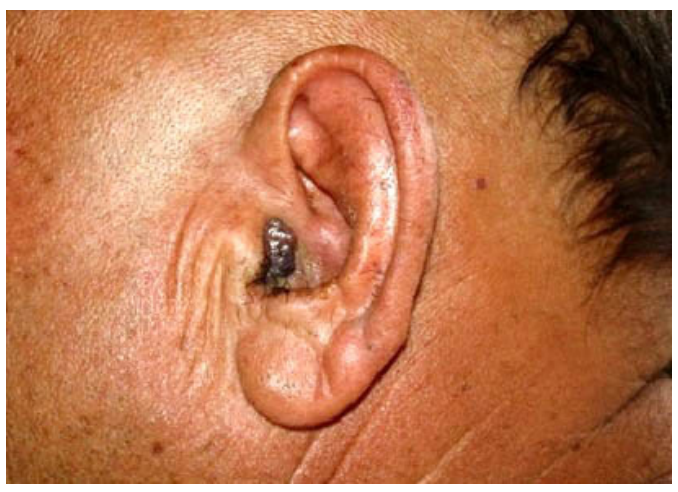

Figura 1. Macrofotografía que muestra la lesión tumoral protuberante, oclusiva y pigmentada, que obstruye el conducto auditivo externo. 
torio del tumor fue de melanoma de CAE y múltiples adenopatías metastásicas de este tipo de neoplasia.

Como reporte por parte de patología se obtuvo un melanoma infiltrante con crecimiento vertical con formación de núcleos celulares, organoides con células epiteloides, que se extiende hasta la dermis profunda, no ulcerado, nivel de Clark III, índice de Breslow >5 mm, índice mitótico bajo de 5 nidos por campo. Invasión vascular superficial de la dermis papilar, hiperplasia epidérmica periférica entre los nidos tumorados, bordes libres de resección quirúrgica. Con confirmación de inmunorreacción intensamente positiva para el HMB-45, IHQ-20 X (figuras 2 a 5).

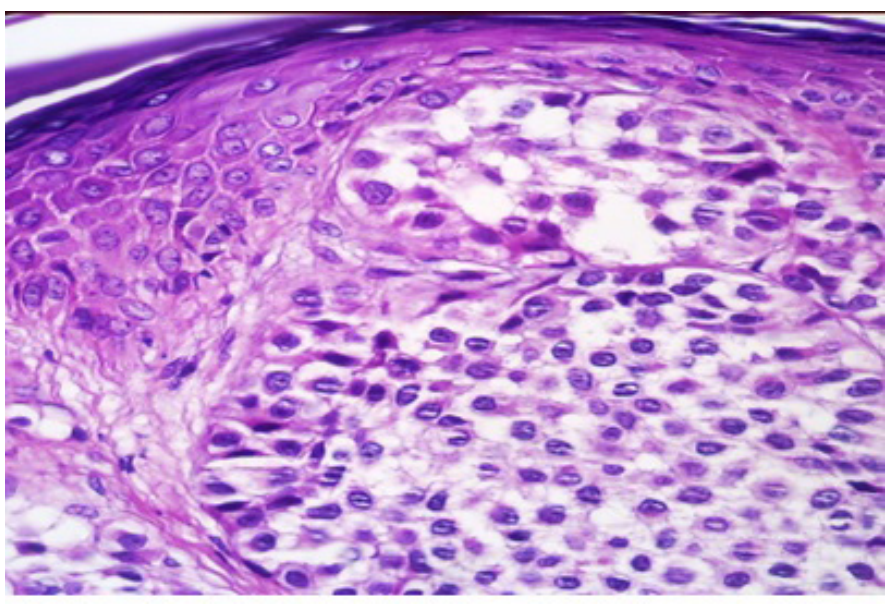

Figura 2. Microfotografía de un campo a mayor aumento de la lesión. Obsérvese la presencia de células pleomórficas y ligeramente anaplásicas. $\mathrm{H} / \mathrm{E}-40 \mathrm{X}$.

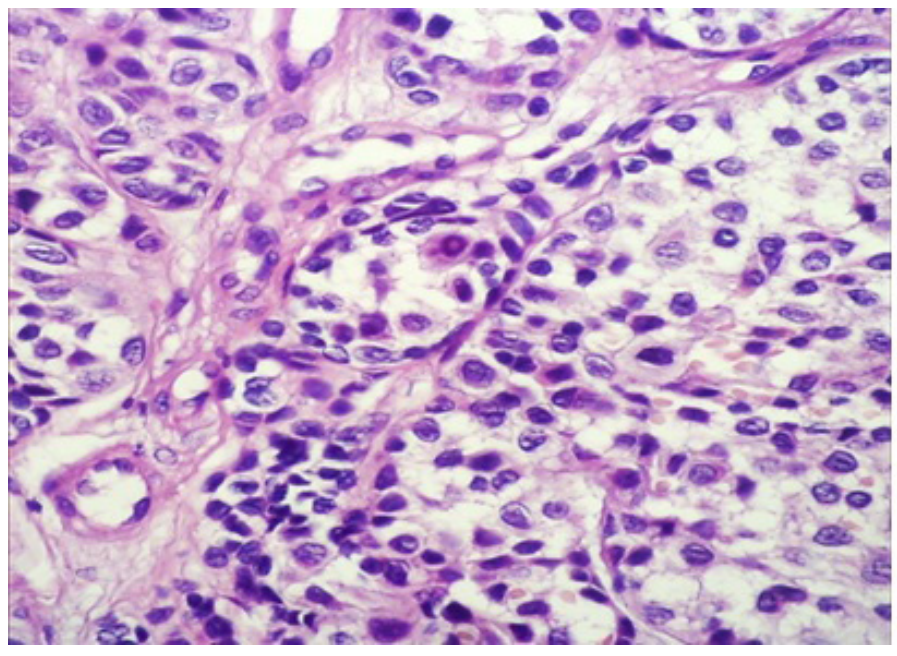

Figura 3. Campo histológico de otra área del tumor, que no muestra el pigmento melánico típico y sí un patrón alveolar. H/E-40 X. 


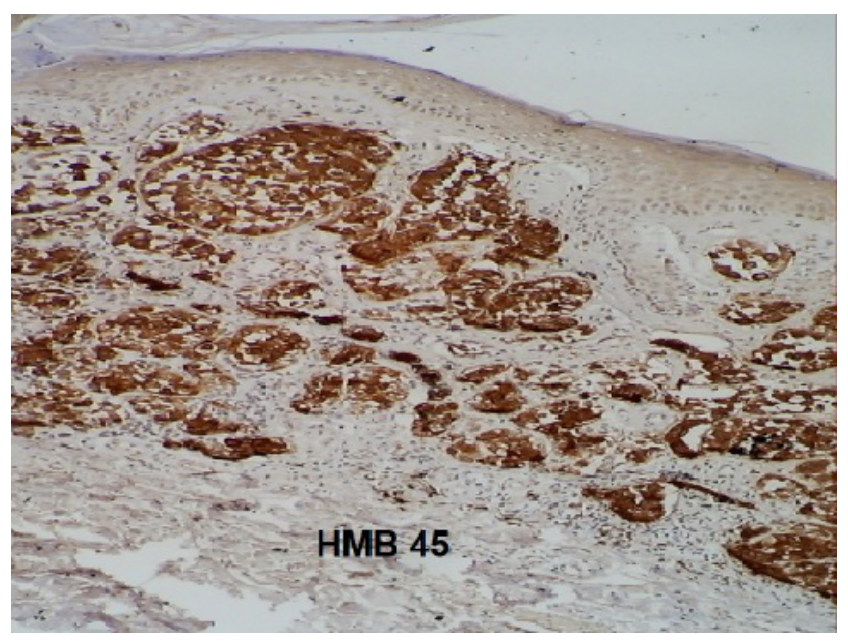

Figura 4. Inmunorreacción intensamente positiva para el HMB-45. IHQ-20 X.

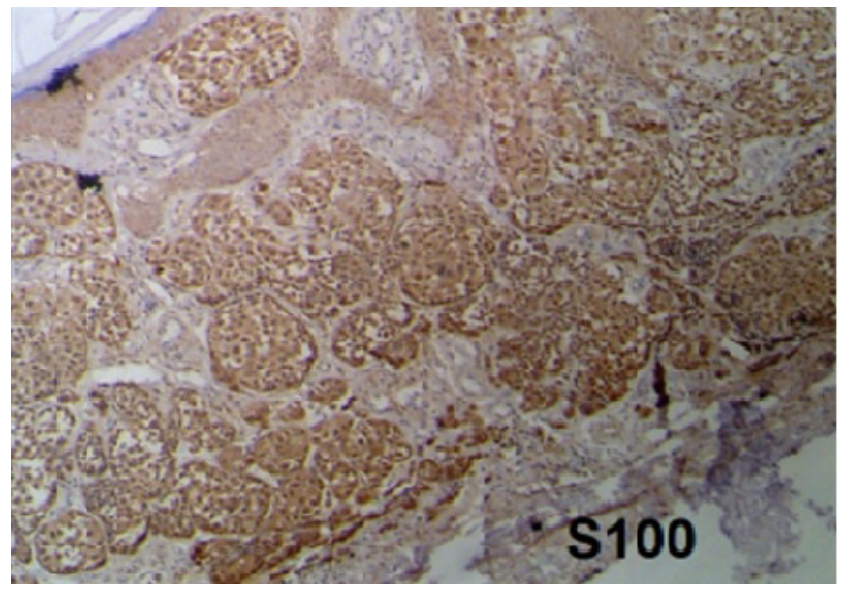

Figura 5. Reactividad intensamente positiva al anticuerpo S-100. IHQ-20 X.

El enfermo fue trasladado al Hospital Oncológico Madame Curie, donde se cumplió el tratamiento con radioterapia en múltiples sesiones, con una dosis total de 2400 CGy. Después de seis meses de seguimiento periódico, el paciente ha mejorado. 


\section{DISCUSIÓN}

El melanoma maligno del CAE en una entidad rara y, sobre todo, en regiones no expuestas a la radiación solar ${ }^{(2,4)}$. El diagnóstico diferencial de estos tumores debe hacerse con entidades menos frecuentes de esta localización, como el carcinoma epidermoide, los paragangliomas, las enfermedades granulomatosas y el colesteatoma ${ }^{(4,5)}$.

Con frecuencia, los pacientes presentan otalgia de larga evolución, otorrea, secreción sanguinolenta y pérdida de la audición. Estos síntomas son bastante inespecíficos y pueden asociarse a infecciones crónicas del CAE. Otra presentación de la lesión es en forma de pólipo auditivo. Además, se eleva un alto índice de sospecha cuando una lesión pigmentada cambia de color, se agranda rápidamente o se ulcera con mala cicatrización ${ }^{(2)}$.

La biopsia es el método diagnóstico de elección, que permite llegar al diagnóstico de certeza, como ocurrió en este paciente. En ocasiones, fue necesaria la confirmación con técnicas de inmunohistoquímica (HBM-45 y S-100) en este enfermo ${ }^{(4)}$. Los criterios histopatológicos de mayor valor pronóstico son: el subtipo histológico, el grosor y la presencia de ulceración ${ }^{(4)}$. Las zonas preauricular y auricular son consideradas regiones de peor pronóstico dentro de la región de cabeza y cuello (4). Dada la capacidad de diseminación sistémica de los melanomas, es necesario en el estudio preoperatorio descartar la presencia de metástasis hepáticas, pulmonares y óseas ${ }^{(6)}$. En el momento del diagnóstico, existe frecuentemente diseminación del tumor a la parótida o a las cadenas linfáticas de drenaje, siendo los ganglios parotídeos y preauriculares la primera manifestación ${ }^{(4-6)}$, como ocurrió en este caso. Algunos autores ${ }^{(2,6)}$ han señalado que, en el momento del diagnóstico, del 30\% al 40\% de los pacientes con esta entidad presentaban adenopatías sospechosas de infiltración metastásica. El pronóstico de los melanomas en esta localización es pobre y es peor que las localizadas en el pabellón auricular. Algunos autores muestran una supervivencia a los 5 años del 47\%, que pudiera deberse al retraso en el diagnóstico ${ }^{(7-9)}$.

Los autores y las guías de manejo recomiendan la opción quirúrgica como primera aproximación terapéutica ${ }^{(2,5,7)}$. En tumores pequeños se recomienda la resección en bloque, mientras que en tumores muy extensos se requiere escisiones amplias y agresivas, con asociación de radioterapia, si fuese necesario, como en este caso ${ }^{(10)}$. Se han informado varios métodos que utilizan colgajos libres. Estos métodos previenen la estenosis, la infección crónica y la cicatrización de heridas. Sin embargo, se prefiere el injerto de piel debido a la simplicidad de la técnica ${ }^{(2)}$.

Ante la presencia de metástasis se sugiere quimioterapia, utilizando la dacarbazina como único medicamento o en combinación con otros fármacos. En cuanto a la bioquimioterapia, se menciona el interferón alfa para la enfermedad metastásica cuando hay alto riesgo de recaída, especialmente en quienes se detecta compromiso ganglionar, y la interleucina 2 endovenosa en altas dosis ${ }^{(11)}$. En los últimos años se han aprobado nuevas inmunoterapias para el tratamiento del melanoma. Una de estas es el ipilimumab, que actúa disminuyendo la inmunotolerancia y es capaz de favorecer la involución del tumor. Por otro lado, se reportaron medianas de sobrevida superiores a los 16 meses con los anticuerpos monoclonales humanizados pembrolizumab y nivolumab. Para aquellos melanomas que presentan una mutación de BRAF, el vemurafenib, el dabrafenib y el trametinib han demostrado buenos resultados ${ }^{(12)}$.

\section{CONCLUSIONES}

El melanoma maligno primario puede ocurrir en cualquier superficie de la piel que posea meloncitos. Presentamos el caso de un paciente con melanoma del CAE, cuya localización es muy infrecuente. Esta enfermedad se debe sospechar ante una lesión pigmentada que presente cambios de coloración, ulceraciones o hemorragias, y es de muy mal pronóstico. El diagnóstico se realiza mediante estudios histopatológicos, que, en ocasiones, requieren estudios de inmunohistoquímica. Existe una fuerte asociación entre la exposición solar prolongada y esta entidad. La opción quirúrgica es la principal terapéutica asociada a la radio y quimioterapia, si fuese necesario.

\section{REFERENCIAS}

1. Landau DC, Caruso TA, Garay IS, Kurpis M, Ruiz A. Melanoma in external auditory canal: regarding a case report. Med Cutan Iber Lat Am. 2018;46(2):120-23.

2. Chebrolu G, Prasanna K, Ravikumar A, Leena J, Swaminathan R. Malignant Melanoma of the External Auditory Canal. J Clin Diagn Res. 2014;8(8):FDo4-FDo6. 
3. Weissman JL. A pain in the ear: The radiology of otalgia. ARJNR Am J Neuroradiol. 2005;18(9):164151.

4. O'Brien CJ, Coates AS, Petersen-Schaefer K, Shannon K, Thompson J, Milton GW, et al. Experience with 998 cutaneous melanomas of the head and neck over 30 years. Am J Surg. 1991;162(4):310-4.

5. Langman A, Yarington C, Patterson S. Malignant melanoma of the external auditory canal. Otolaryngol Head Neck Surg. 1996;114(4):645-8.

6. Amando García L, Suarez Nieto C, Madrigal Rubiates B, García García J. Melanoma del conducto auditivo externo. Acta Otorrinolaringol Esp. 2003;54:89-93.

7. Alex AC, Krag DN, Harlow SP, Meijer S, Loggie BW, Kuhn J, et al. Localization of regional lymph nodes in melanomas of the head and neck. Arch Otolaryngol Head Neck Surg. 1998;124(2):135-40.

8. Davidson A, Hellquist HB, Villman H, Westman G. Malignant melanoma of the ear. J Laryngol Otol. 1993;107:798-802.

9. Cole DJ, Mackay GJ, Walker BF, Wooden WA, Murray DR, Coleman JJ 3rd. Melanoma of the external ear. J Surg Oncol. 1992;50(2):110-4.

10. Ang KK, Byers RM, Peters LT, Moar MH, Wendt CD, Morrison WU, et al. Regional radiotherapy as adjuvant treatment for head and neck malignant melanoma. Arch Otolaryngol Head Neck Surg. 1990;116(2):169-72.

11. Markovic SN, Erickson LA, Rao RD, Weenig RH, Pockaj BA, Bardia A, et al. Malignant melanoma in the 21st century, part 2: staging, prognosis, and treatment. Mayo Clin Proc. 2007;82(4):490-513.

12. Droppelmann MN, León RA, Goñi EI, González DH, Domínguez CF, Camus AM, et al. Nuevas terapias sistémicas para el tratamiento del melanoma. Rev Chil Cir. 2016;68(1):81-6. 